\title{
Epidemiological statistics of congenital thumb duplication in the Chinese population
}

\author{
Yingling Yao ${ }^{1,2+}$, Haolin Zhou ${ }^{3+}$, Lianyong $\mathrm{Li}^{3^{*}}$ (D) and Guoxin Nan ${ }^{1,2^{*}}$ (D)
}

\begin{abstract}
Background: Thumb duplication is a very common congenital malformation. This study describes and compares the phenotypic manifestations of polydactyly between southwest and northeast China. However, previous studies had a limited sample size. Therefore, this study used a large sample.
\end{abstract}

Methods: A total of 3549 well-characterized thumb duplication cases were divided into group A (southwest China) and group B (northeast China).

Results: In group A and group B, the left-to-right ratio was 1:1.5 and 1:1.75, respectively, and the female-to-male ratio was $1: 1.5$ and $1: 1.58$, respectively.

Conclusions: There were no significant differences in gender distribution or the distribution of left and right polydactyly between the two groups, but the distribution of bilateral polydactyly was significantly different.

Keywords: Thumb polydactyly, Southwest China, Northeast China, Thumb duplication, Wassel classification

\section{Introduction}

Thumb duplication is a very common congenital malformation. Preaxial polydactyly is the most common duplication in Caucasian and Asian populations, and it occurs in 0.81.4 cases per 1000 births [1]. Abnormal expression of morphogens, such as Hox genes, bone morphogenic proteins, LMBR1, Gli-3, and increased duplication of ZRS region has been associated with thumb duplication [2-5].The Wassel system, which was developed in 1969, has become the

\footnotetext{
*Correspondence: loyo_Idy@163.com; ngx1215@163.com

†Yingling Yao and Haolin Zhou contributed equally to this work and should be considered co-first authors.

${ }^{3}$ Department of Pediatric Orthopedics, Shengjing Hospital of China Medical University, No.36 Sanhao Street, Heping District, Shenyang City 110004, China ${ }^{1}$ Ministry of Education Key Laboratory of Child Development and Disorders, National Clinical Research Center for Child Health and Disorders

(Chongqing), China International Science and Technology Cooperation Base of Child Development and Critical Disorders, Stem Cell Biology and Therapy Laboratory, Chongqing Key Laboratory of Pediatrics, Children's Hospital of Chongqing Medical University, Chongqing 400014, China

Full list of author information is available at the end of the article
}

universal classification system for thumb duplication due to its simplicity [1]. Type IV thumb duplication is the most common type, and it is followed by type II and then type VII [5-7].

Several series have reported the distribution of different types of thumb polydactyly in domestic and foreign populations. However, there is no large sample study of polydactyly in northeast and southwest China. There are differences in climate, topography, ethnicity, economic level, diet, and medical level in northeast and southwest China. The northeast is mainly a plain with a cold climate, while the southwest is dominated by a mountainous plateau basin with a subtropical monsoon climate and a large number of ethnic minorities. Therefore, the purpose of this study is to describe the epidemiological characteristics of thumb duplication based on a statistical analysis of the Chinese population and to elucidate whether the clinical and epidemiological characteristics of thumb duplication

(c) The Author(s). 2021 Open Access This article is licensed under a Creative Commons Attribution 4.0 International License, which permits use, sharing, adaptation, distribution and reproduction in any medium or format, as long as you give appropriate credit to the original author(s) and the source, provide a link to the Creative Commons licence, and indicate if changes were made. The images or other third party material in this article are included in the article's Creative Commons licence, unless indicated otherwise in a credit line to the material. If material is not included in the article's Creative Commons licence and your intended use is not permitted by statutory regulation or exceeds the permitted use, you will need to obtain permission directly from the copyright holder. To view a copy of this licence, visit http://creativecommons.org/licenses/by/4.0/ The Creative Commons Public Domain Dedication waiver (http://creativecommons.org/publicdomain/zero/1.0/) applies to the data made available in this article, unless otherwise stated in a credit line to the data. 
in southwest China differ from its clinical and epidemiological characteristics in northeast China.

\section{Methods}

Patients with thumb duplication were identified between 2012 and 2019 at Children's Hospital of Chongqing Medical University (southwest China) and China Medical University (northeast China). The diagnosis of thumb duplication is done by two or more clinicians. The thumb duplication classification is established by two doctors after independent judgment; If there is a disagreement, the typing result is discussed together after a third doctor has made a judgment. The classified according to the Wassel classification (Table 1).

A total of 3549 cases from different families with thumb duplication were included in this study.

The group with thumb duplication recruited from the hospital in southwestern China was designated as group $\mathrm{A}$, and the group with thumb duplication recruited from the hospital in northeastern China was designated as group B. Compare the gender and left-right differences of thumb duplication patients in the two regions.

Statistical analysis was performed using SPSS 23.0 software, qualitative data were described by percentages; count data were expressed as rates or composition ratios, and differences between groups were analyzed using the $\chi^{2}$ test. $P<0.05$ (two-sided).

This study has been approved by the Institutional Review Board (IRB) of our hospital.

\section{Results}

In group A, there were 2463 cases of thumb duplication. Of these, 333 were cases of bilateral thumb duplication. A positive family history was recorded in 160 cases. In total, there were 1492 males, who accounted for 60.6\% of the population, and 971 females, who accounted for $39.4 \%$ of the population. The male-to-female ratio was $1.5: 1$, and the left-to-right thumb duplication ratio was 1:1.5 (1121 with left thumb duplication and 1675 with right thumb duplication). In case of unilateral thumb duplication, polydactyly was observed in 1274 males and 857 females (Table 2).

Table 1 Classification of thumb polydactyly used in the current study

\begin{tabular}{ll}
\hline Wassel I & Bifid distal phalanx \\
\hline Wassel II & Duplicated distal phalanx \\
Wassel III & Bifid proximal phalanx \\
Wassel IV & Duplicated proximal phalanx \\
Wassel V & Bifid metacarpal \\
Wassel VI & Duplicated metacarpal \\
Wassel VII & Thumb duplication with triphalangism \\
Rudimentary & Small appendage with a narrow pedicle \\
\hline
\end{tabular}

Table 2 Distribution of thumb polydactyly according to gender (group A)

\begin{tabular}{llll}
\hline Sex & $\mathbf{n}$ & Unilateral & Bilateral \\
\hline Male & 1492 & $1274(85.4)$ & $218(14.6)$ \\
Female & 971 & $857(88.3)$ & $114(11.7)$ \\
$X 2$ & & 4.157 & \\
$P$ & & 0.041 & \\
\hline
\end{tabular}

The exclusion criteria of classification are as follows: [1] re-admission to the hospital for lesions and deformities, [2] imaging done outside the hospital without imaging data from our hospital. The types of polydactyly observed in the current study cohort are shown in Table 3. There were 2463 cases of thumb duplication, out of which 200 (8.0\%) were excluded and 2263 (2562 fingers) were included. Among the included cases, 238 (11\%) did not fit the classic Wassel types, and 5 cases did not fit into any of the Rotterdam classification types. Type IV thumb duplication accounted for the overwhelming majority of the patients (35.3\%, 905/2562). Further, type II thumb duplication was observed in $13.7 \%$ (351/2562) of the cases, type $\mathrm{V}$ in $9.5 \%$ (243/2562) of the cases, and type VII and type I in $9.1 \%$ (233/2562) and $2.6 \%(67 / 2562)$ of the cases, respectively (Table 3). So, the total of 2025 cases (2279 fingers) of thumb duplication has been classified by Wassel. The present findings are compared with those from other studies at home and in other countries in Table 4. Further, as shown in Table 5, there was no significant difference in the distribution of the different types with regard to $\operatorname{sex}\left(X^{2}=12.146, P=0.096\right)$ or affected side $\left(X^{2}=\right.$ $11.134, P=0.133)$. Bilateral rudimentary thumb duplication was more common than unilateral rudimentary thumb duplication $(P<0.05$, Table 5$)$.

In group B, out of 1086 patients, 665 were male and 421 were female. Of these, 89 had bilateral thumb polydactyly. The male-to-female ratio was $1.58: 1$, and the left-to-right polydactyly ratio was 1:1.75 (362 with thumb polydactyly on the left hand and 635 with thumb polydactyly on the right).

There were no significant differences between groups $A$ and $B$ in terms of either gender distribution or leftright sides $(P>0.05)$.

The distribution of bilateral polydactyly was significantly different between the two groups $\left(X^{2}=20.395\right.$, $P<0.001$, Table 6).

\section{Discussion}

This study analyzed and compared the demographic and clinical characteristics of thumb duplication between southwestern and northeastern China. The obtained findings and the results obtained from the large sample size of this study could be a valuable resource for comparing the features of thumb duplication in the Chinese 
Table 3 Distribution of the types of thumb polydactyly in 2562 hands (group A)

\begin{tabular}{|c|c|c|c|c|c|c|c|c|}
\hline Wassel type & Male & Female & Left & Right & Unilateral & Bilateral & Total & $(\%)$ \\
\hline \multicolumn{9}{|c|}{ (Number of hands) } \\
\hline I & 41 & 26 & 18 & 49 & 52 & 15 & 67 & 2.6 \\
\hline II & 194 & 157 & 138 & 213 & 295 & 56 & 351 & 13.7 \\
\hline III & 127 & 89 & 91 & 125 & 178 & 38 & 216 & 8.4 \\
\hline IV & 580 & 325 & 348 & 557 & 731 & 174 & 905 & 35.3 \\
\hline $\mathbf{v}$ & 149 & 94 & 90 & 153 & 177 & 66 & 243 & 9.5 \\
\hline VI & 62 & 29 & 42 & 49 & 63 & 28 & 91 & 3.6 \\
\hline VII & 136 & 97 & 91 & 142 & 180 & 53 & 233 & 9.1 \\
\hline Rudimentary & 111 & 62 & 80 & 93 & 95 & 78 & 173 & 6.8 \\
\hline Others & 168 & 115 & 113 & 170 & 193 & 90 & 283 & 11.0 \\
\hline Total & 1568 & 994 & 1011 & 1551 & 1964 & 598 & 2562 & 100 \\
\hline
\end{tabular}

population with those in other populations, and could promote research on the phenotypic variability of this condition and factors related to it.

This study showed that there were no significant differences between northeast and southwest China in terms of either gender distribution or left-right sides, but the distribution of bilateral polydactyly was significantly different. However, there are differences between the research data of other countries and the research results of this paper. For example, Ozalp et al. in Turkey and Islam et al. in Japan reported a male-to-female ratio of 1:1, and the results reported by Su et al. in China and Naasan et al. in Hong Kong both showed that the maleto-female ratio was $2: 1$ and the left-to-right thumb polydactyly ratio was $1: 1.5$ [8-11]. The difference in the ratios indicates that the incidence rates in other countries in Europe and Asia are different from those in China. This difference may be attributable to differences in race, environment, economic level, social medical security, diet, and national health awareness. Additionally, the differences between Turkey, Japan, and China are mainly attributed to differences in ethnicity. However, as this dataset is also different from other domestic datasets, it is possible that other factors, such as environment, diet, geography, and sample size, are responsible for the difference in incidence.

In all races, Wassel type IV seems to be the most common, while Wassel type I seems to be the least common thumb polydactyly. However, there are differences in the specific proportions of each type reported. The results of this study in southwestern China show that type IV accounts for $39.7 \%$ of the affected population, while the proportion reported in another domestic study by $\mathrm{Su}$ et al., the Middle East study by Al-Qattan et al., the Japan study by Islam et al., and the UK study by Naasan et al. show that type IV accounts for $29.6 \%, 35 \%, 33.6 \%$, and $20.9 \%$ of the respective populations [10-12].This is different from our statistical results and may be associated with the sample size. This group of data has a large sample size, similar to the previous reports at home and abroad. At present, the classification is mainly based on imaging. However, for infants and young children, it is often difficult to distinguish the epiphysis from the ossification center by radiography, and this affects the classification of type IV, V, and VII thumb polydactyly. In the case data from this group, difficulty in classification

Table 4 Comparison of the proportions of Wassel types across several domestic and international studies

\begin{tabular}{|c|c|c|c|c|c|c|c|c|c|c|}
\hline \multirow[t]{2}{*}{ Wassel type } & \multicolumn{2}{|l|}{ Our data } & \multicolumn{2}{|c|}{ China, Su et al. } & \multicolumn{2}{|c|}{ The Middle East, Al-Qattan et al. } & \multicolumn{2}{|c|}{ Japan, Islam et al. } & \multicolumn{2}{|c|}{ The UK, Naasan A et al. } \\
\hline & Number & $\%$ & Number & $\%$ & Number & $\%$ & Number & $\%$ & Number & $\%$ \\
\hline I & 67 & 2.9 & 5 & 1.7 & 1 & 0.5 & 6 & 3.8 & 2 & 4.7 \\
\hline$\|$ & 351 & 15.4 & 43 & 14.5 & 21 & 9.5 & 36 & 22.8 & 13 & 30.2 \\
\hline III & 216 & 9.5 & 16 & 5.4 & 31 & 14.1 & 8 & 5.1 & 8 & 18.6 \\
\hline IV & 905 & 39.7 & 88 & 29.6 & 77 & 35 & 53 & 33.6 & 9 & 20.9 \\
\hline V & 243 & 10.7 & 23 & 7.8 & 13 & 5.9 & 10 & 6.3 & 4 & 9.3 \\
\hline $\mathrm{Vl}$ & 91 & 4.0 & 20 & 6.7 & 31 & 14.1 & 11 & 6.9 & 4 & 9.3 \\
\hline VII & 233 & 10.2 & 83 & 27.9 & 28 & 12.7 & 11 & 6.9 & 3 & 7.0 \\
\hline Rudimentary & 173 & 7.6 & 19 & 6.4 & 18 & 8.2 & 23 & 14.6 & & \\
\hline Total & 2279 & $100 \%$ & 297 & $100 \%$ & 220 & $100 \%$ & 158 & $100 \%$ & 43 & $100 \%$ \\
\hline
\end{tabular}


Table 5 Phenotypic characteristics of thumb duplication in group A

\begin{tabular}{|c|c|c|c|c|c|c|c|c|c|c|c|c|c|c|c|c|c|c|}
\hline \multirow[t]{2}{*}{ Variables } & \multirow{2}{*}{$\begin{array}{l}\text { Total } \\
\text { N }\end{array}$} & \multicolumn{2}{|l|}{ I } & \multicolumn{2}{|l|}{ II } & \multicolumn{2}{|l|}{ III } & \multicolumn{2}{|l|}{ IV } & \multicolumn{2}{|l|}{ v } & \multicolumn{2}{|l|}{ VI } & \multicolumn{2}{|l|}{ VII } & \multicolumn{2}{|c|}{ Rudimentary } & \multirow[t]{2}{*}{$P$} \\
\hline & & $\mathrm{N}$ & $\%$ & $\mathrm{~N}$ & $\%$ & $\mathrm{~N}$ & $\%$ & $\mathrm{~N}$ & $\%$ & $\mathrm{~N}$ & $\%$ & $\mathrm{~N}$ & $\%$ & $\bar{N}$ & $\%$ & $\mathrm{~N}$ & $\%$ & \\
\hline \multicolumn{19}{|l|}{ Sex } \\
\hline Male & 1400 & 41 & 2.9 & 194 & 13.9 & 127 & 9.1 & 580 & 41.4 & 149 & 10.6 & 62 & 4.4 & 136 & 7.9 & 111 & 7.9 & 0.096 \\
\hline Female & 879 & 26 & 3.0 & 157 & 17.9 & 89 & 10.1 & 325 & 37.0 & 94 & 10.7 & 29 & 3.3 & 97 & 11.0 & 62 & 7.1 & \\
\hline \multicolumn{19}{|l|}{ Laterality } \\
\hline Unilateral & 1771 & 52 & 2.9 & 295 & 16.7 & 178 & 10.1 & 731 & 41.3 & 177 & 10.0 & 63 & 3.6 & 180 & 10.2 & 95 & $5.4^{\mathrm{a}}$ & $<0.001^{b}$ \\
\hline Bilateral & 508 & 15 & 3.0 & 65 & 11.0 & 38 & 7.5 & 174 & 34.3 & 66 & 13.0 & 28 & 5.5 & 53 & 10.4 & 78 & $15.4^{\mathrm{a}}$ & \\
\hline \multicolumn{19}{|l|}{ Sidedness } \\
\hline Left & 898 & 18 & 2.0 & 138 & 15.4 & 91 & 10.1 & 348 & 38.8 & 90 & 10.0 & 42 & 4.7 & 91 & 10.1 & 80 & 8.9 & 0.133 \\
\hline Right & 1381 & 49 & 3.5 & 213 & 15.4 & 125 & 9.1 & 557 & 40.3 & 153 & 11.1 & 49 & 3.5 & 142 & 10.3 & 93 & 6.7 & \\
\hline
\end{tabular}

${ }^{a}$ Significant for the rudimentary group compared with other Wassel types

${ }^{\mathrm{b}}$ Significant difference between groups

before surgery was usually solved by intraoperative classification.

Although the Wassel system represents a universal system of classification of thumb duplication because of its simplicity, it does not represent all types of thumb duplication [13]. Therefore, others have attempted to improve upon the Wassel system, including BuckGramcko, Upton, and Flatt, with the Rotterdam classification [1].A study by Dijkman et al. compared the reliability of the Wassel and Rotterdam classifications [7]. Out of a study population of 520 cases, only $60 \%$ could be classified using the Wassel classification, compared with $100 \%$ using the Rotterdam classification. However, Su et al. showed that only $8.6 \%$ of hands could not be classified with the Wassel classification system [11]. In comparison, in the present study, a total of 238 fingers (11\%) did not fit into the classic Wassel types. A study by $\mathrm{Hu}$ et al. also showed that adding a hypoplastic subtype to the Wassel-Flatt can classify most of previously unclassifiable thumbs [14]. Therefore, it is necessary to propose a new classification method to supplement the existing Wassel classification.

In this regard, Gao et al. report a new classification that can be used to comprehensively describe the clinical features of the terminal phalanx in congenital thumb duplication and the surgical procedure that can be adopted for each type with satisfactory results [15].Additionally, Chung et al. developed a new classification method that is more correlated with the therapeutic approach [16]. Moreover, He et al. proposed atypical Wassel type VI and formulated corresponding treatment plans, with satisfactory treatment results and reduced complications [17]. For special cases that cannot be classified into the Wassel classification system, it is necessary to increase the sample size, to summarize the pathological and anatomical characteristics, and to further classify them to overcome the shortcomings of the existing Wassel typing methods and guide clinical treatment.

To conclude, this study could enhance our understanding of the distribution of thumb duplication types based on sex, affected side, and genetic inheritance in the Chinese population. Additionally, the findings are valuable in terms of exploring the prevalence of polydactylyassociated congenital anomalies in the Chinese population by means of epidemiological information on thumb duplication. However, this study only has case data from Southwest China and Northeast China and does not include cases across the country. If conditions permit, we will conduct a multi-center study to collect more cases.

Table 6 Phenotypic characteristics of thumb duplication between group A and group B

\begin{tabular}{|c|c|c|c|c|c|c|c|c|}
\hline \multirow[t]{2}{*}{ Variables } & \multicolumn{2}{|l|}{ Total } & \multicolumn{2}{|l|}{$\mathrm{Aa}$} & \multicolumn{2}{|l|}{$\mathrm{Bb}$} & \multirow[t]{2}{*}{$x^{2}$} & \multirow[t]{2}{*}{$P$} \\
\hline & Number & $\%$ & Number & $\%$ & Number & $\%$ & & \\
\hline \multicolumn{9}{|l|}{ Sex } \\
\hline Male & 2157 & 60.8 & 665 & 61.2 & 1492 & 60.6 & 0.137 & 0.712 \\
\hline Female & 1392 & 39.2 & 421 & 38.8 & 971 & 39.4 & & \\
\hline \multicolumn{9}{|l|}{ Laterality } \\
\hline Left & 1150 & 32.4 & 362 & 33.3 & 788 & 32.0 & 0.618 & 0.432 \\
\hline Right & 1977 & 55.7 & 635 & 58.5 & 1342 & 54.5 & 4.851 & 0.028 \\
\hline Bilateral & 422 & 11.9 & 89 & 8.2 & 333 & 13.5 & 20.395 & $<0.001 c$ \\
\hline
\end{tabular}

${ }^{a}$ Thumb duplication features of the group $A$

${ }^{b}$ Thumb duplication features of the group $B$

'Significant for each group compared with their controls 


\section{Acknowledgements}

None

\section{Authors' contributions}

Guoxin Nan and Lianyong Li contributed to the study conception and design. Yingling Yao and Haolin Zhou contributed to the acquisition of the data. Haolin Zhou contributed to the analysis and interpretation of the data. Yingling Yao drafted the manuscript, and Guoxin Nan and Lianyong Li revised it. Yingling Yao and Haolin Zhou contributed equally to this work and should be considered co-first authors. Guoxin Nan and Lianyong Li are the co-corresponding authors. The authors read and approved the final manuscript.

\section{Funding}

No funding was received for conducting this study.

\section{Availability of data and materials}

The datasets used and/or analyzed during the current study are available from the corresponding author on reasonable request.

\section{Declarations}

\section{Ethics approval and consent to participate}

This study has been approved by the Children's Hospital of Chongqing Medical University (no. 2020-29). Informed consent was obtained from all individual patients included in the study

\section{Consent for publication}

Patients signed informed consent regarding publishing their data and photographs, and manuscript is approved by all authors for publication.

\section{Competing interests}

The authors declare that they have no competing interests.

\section{Author details}

${ }^{1}$ Ministry of Education Key Laboratory of Child Development and Disorders, National Clinical Research Center for Child Health and Disorders

(Chongqing), China International Science and Technology Cooperation Base of Child Development and Critical Disorders, Stem Cell Biology and Therapy Laboratory, Chongqing Key Laboratory of Pediatrics, Children's Hospital of Chongqing Medical University, Chongqing 400014, China. ${ }^{2}$ Department of Orthopaedics, Children's Hospital of Chongqing Medical University,

Chongqing, China. ${ }^{3}$ Department of Pediatric Orthopedics, Shengjing Hospital of China Medical University, No.36 Sanhao Street, Heping District, Shenyang City 110004, China.

Received: 31 March 2021 Accepted: 17 June 2021

Published online: 09 August 2021

References

1. Wyhe RDV, Trost JG, Koshy JC, Pederson WC. The duplicated thumb: a review. Seminars Plastic Surg. 2016;30(04):181-8. https://doi.org/10.1055/s0036-1593736.

2. Dautel G, Perrin P. Use of an axial flap to increase the girth of Wassel IV thumb reconstructions. J Hand Surg A. 2015;40(7):1327-32. https://doi.org/1 0.1016/j.jhsa.2015.02.032

3. Xu J, Wu J, Teng $X$, Cai L, Yuan $H$, Chen $X$, et al. Large duplication in LMBR1 gene in a large Chinese pedigree with triphalangeal thumb polysyndactyly syndrome. Am J Med Genet A. 2020;182(9):2117-23. https://doi.org/10.1 002/ajmg.a.61757.

4. Shi $L$, Huang $H$, Jiang $Q$, Huang $R$, Fu W, Mao $L$, et al. Sub-exome target sequencing in a family with syndactyly type iv due to a novel partial duplication of the Imbr1 gene: first case report in fujian province of china. Front Genet. 2020;11:130. https://doi.org/10.3389/fgene.2020.00130.

5. Wang B, Diao Y, Liu Q, An H, Ma R, Jiang G, et al. An increased duplication of ZRS region that caused more than one supernumerary digits preaxial polydactyly in a large Chinese family. Sci Rep. 2016;6(1):38500. https://doi. org/10.1038/srep38500.

6. Perez-Lopez LM, la Iglesia DG, Cabrera-Gonzalez M. Radial polydactyly. What's new? Curr Pediatr Rev. 2018:14(2):91-6. https://doi.org/10.2174/15733 96314666180124102012.
7. Dijkman RR, van Nieuwenhoven CA, Selles RW, Habenicht R, Hovius SER. A multicenter comparative study of two classification systems for radial polydactyly. Plast Reconstr Surg. 2014;134(5):991-1001. https://doi.org/10.1 097/PRS.0000000000000590.

8. Ozalp T, Coşkunol E, Ozdemir O. Cift başparmak anomalisi: 72 olgunun değerlendirilmesi [Thumb duplication: an analysis of 72 thumbs]. Acta Orthop Traumatol Turc. 2006;40(5):388-91.

9. Islam S, Oka I, Fujita S. Anatomical study of preaxial polydactyly in 158 hands. Tohoku J Exp Med. 1992;168(3):459-66. https://doi.org/10.1620/ tjem.168.459.

10. Naasan A, Page RE. Duplication of the thumb: a 20-year retrospective review. J Hand Surg Br. 1994;19(3):355-60. https://doi.org/10.1016/0266-7681 (94)90089-2.

11. Su S, Chen ZB, Huang QS. A discussion on the Wassel classification of congenital thumb duplication. Chin J Hand Surg. 2014;30:169-71.

12. Al-Qattan MM. The distribution of the types of thumb polydactyly in a middle eastern population: a study of 228 hands. J Hand Surg Eur Vol. 2010; 35(3):182-7. https://doi.org/10.1177/1753193409352417.

13. Evanson BJ, Hosseinzadeh P, Riley SA, Burgess RC. Radial polydactyly and the incidence of reoperation using a new classification system. J Pediatr Orthop. 2016;36(2):158-60. https://doi.org/10.1097/BPO.0000000000000395.

14. Hu CH, Thompson ER, Agel J, Bauer AS, Moeller AT, Novotny SA, et al. A comparative analysis of 150 thumb polydactyly cases from the could registry using the Wassel-flatt, rotterdam, and chung classifications sciencedirect. J Hand Surg Am. 2020;S0363-5023:30347-6. https://doi.org/1 0.1016/j.jhsa.2020.06.009

15. Gao WY, Wang AY, Ding J. The classification and surgical treatment of the terminal phalanx of congenital thumb duplication. Chin J Plast surg. 2014; 30:330-5.

16. Chung MS, Baek GH, Gong HS, Lee HJ, Kim J, Rhee SH. Radial polydactyly: proposal for a new classification system based on the 159 duplicated thumbs. J Pediatr Orthop. 2013;33(2):190-6. https://doi.org/10.1097/BPO Ob013e3182648ca8.

17. He B, Liu G, Nan G. The anatomy of Wassel type IV-D thumb duplication. J Hand Surg Eur. 2017:42:516-22. https://doi.org/10.1177/1753193417691747.

\section{Publisher's Note}

Springer Nature remains neutral with regard to jurisdictional claims in published maps and institutional affiliations.
Ready to submit your research? Choose BMC and benefit from:

- fast, convenient online submission

- thorough peer review by experienced researchers in your field

- rapid publication on acceptance

- support for research data, including large and complex data types

- gold Open Access which fosters wider collaboration and increased citations

- maximum visibility for your research: over $100 \mathrm{M}$ website views per year

At $\mathrm{BMC}$, research is always in progress.

Learn more biomedcentral.com/submissions 